\title{
Bleeding in COVID Patients: What We Have Understood So Far
}

\author{
Pierleone Lucatelli ${ }^{1}$ (i) $\cdot$ Bianca Rocco $^{1} \cdot$ Pier Giorgio Nardis $^{1} \cdot$ Alessandro Cannavale $^{1}$. \\ Mario Bezzi ${ }^{1} \cdot$ Carlo Catalano $^{1} \cdot$ Mario Corona $^{1}$
}

Received: 21 December 2020/Accepted: 13 January 2021/Published online: 28 January 2021

(C) Springer Science+Business Media, LLC, part of Springer Nature and the Cardiovascular and Interventional Radiological Society of Europe (CIRSE) 2021

Worldwide coronavirus 2019 (COVID-19) pandemic has changed our daily practice as Interventional Radiologists. Central Italy, where our center is located, has been only partially affected by the first pandemic wave, but with the second one impact on the hospitals system's rose.

Rising evidence of severe hypercoagulable state in COVID-19 patients induced to introduce heparin for his anticoagulant action as well as for its anti-viral and antiinflammatory effects [1].

Although thrombotic events represent the most common coagulation disorders, some factors such as thrombocytopenia, hyperfibrinolytic state, consumption of coagulation factors and of course administration of anticoagulant, make COVID-19 patients prone to bleedings [2, 3].

Pierleone Lucatelli

pierleone.lucatelli@gmail.com

Bianca Rocco

biancarocco.br@gmail.com

Pier Giorgio Nardis

p.nardis@gmail.com

Alessandro Cannavale

alessandro.cannavale@hotmail.com

Mario Bezzi

mario.bezzi@gmail.com

Carlo Catalano

carlo.catalano@uniroma1.it

Mario Corona

mario.corona68@gmail.com

1 Vascular and Interventional Radiology Unit, Department of Radiological Oncological and Anatomo-Pathological Sciences, Sapienza University of Rome, Viale Regina Elena, 324, 00161 Rome, Italy
Spontaneous soft tissue hematoma (SSTH) is a wellknown and potentially life-threatening complication of anticoagulation therapy. Interventional radiology management (transarterial embolization, TAE) of patients with SSTH is not codified by international guidelines and is usually offered to patients with hemodynamic instability or failure of medical management [4].

To date, no evidence exists on which COVID-19 patients should be offered TAE. Patients selection is particularly challenging for several reasons: (I) COVID-19 population encompass elderly fragile patients with multiple coagulations disorders; (II) heparin is considered an essential therapy in order to limit COVID-19 vascular lifethreatening complications. In this scenario, the reduction of administered heparin dose or either its suspension has to be balanced with the clinical general conditions and the severity of the bleeding.

In order to understand the appropriate timing of TAE, we share the experience of our Interventional Radiology Unit in the management of COVID-19 bleedings.

Between 15 September and 20 December 2020, we have been consulted for 21 COVID-19 patients undergoing therapy with heparin, for hemoglobin drop (mean $\mathrm{Hb}$ drop: $1.6 \mathrm{~g} / \mathrm{dL}$ ) and evidence of SSTH. A multiphasic contrastenhanced CT showed SSTH located in the rectus sheath (3/ $21)$, iliopsoas muscle $(11 / 21$, with retroperitoneal hemorrhage in 6/10), thoracic wall (4/21) and thight (3/21).

In patients with hemodynamic instability, active blush at $\mathrm{CT}$ scans and $\mathrm{Hb}$ drop $>2 \mathrm{Hb} \mathrm{g} / \mathrm{dL}$, immediate superselective TAE was performed (14/21) permitting bleeding stop and recovery of treated patients in $12 / 14$; two death due to bleeding-related cardiovascular failure occurred in this subgroup $(2 / 14 ; 14.3 \%)$. 


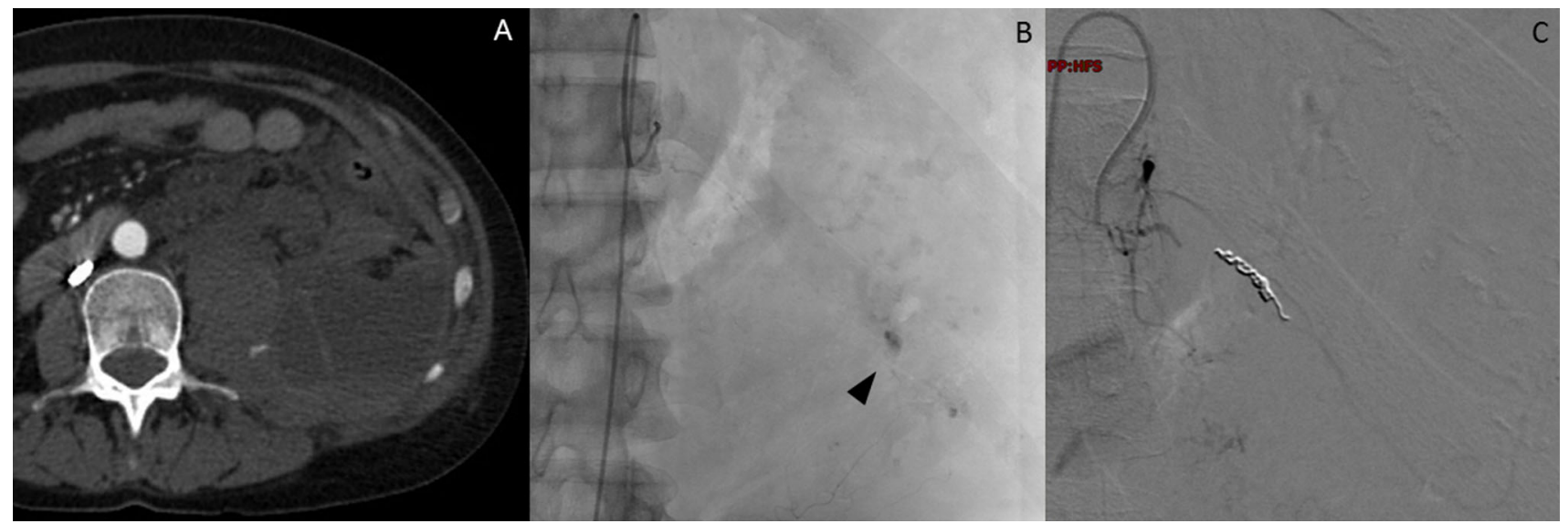

Fig.1 A CT scan of COVID-19 female patient, 61 years old, Hb $7.5 \mathrm{~g} / \mathrm{dL}$ with SSTH of left iliolumbar muscle, associated with retroperitoneal hemorrhage and active blush. B Angiography confirmed numerous sites of bleeding (harrow head). C Embolization with coils alone was performed achieving complete vessel obstruction and bleeding stop
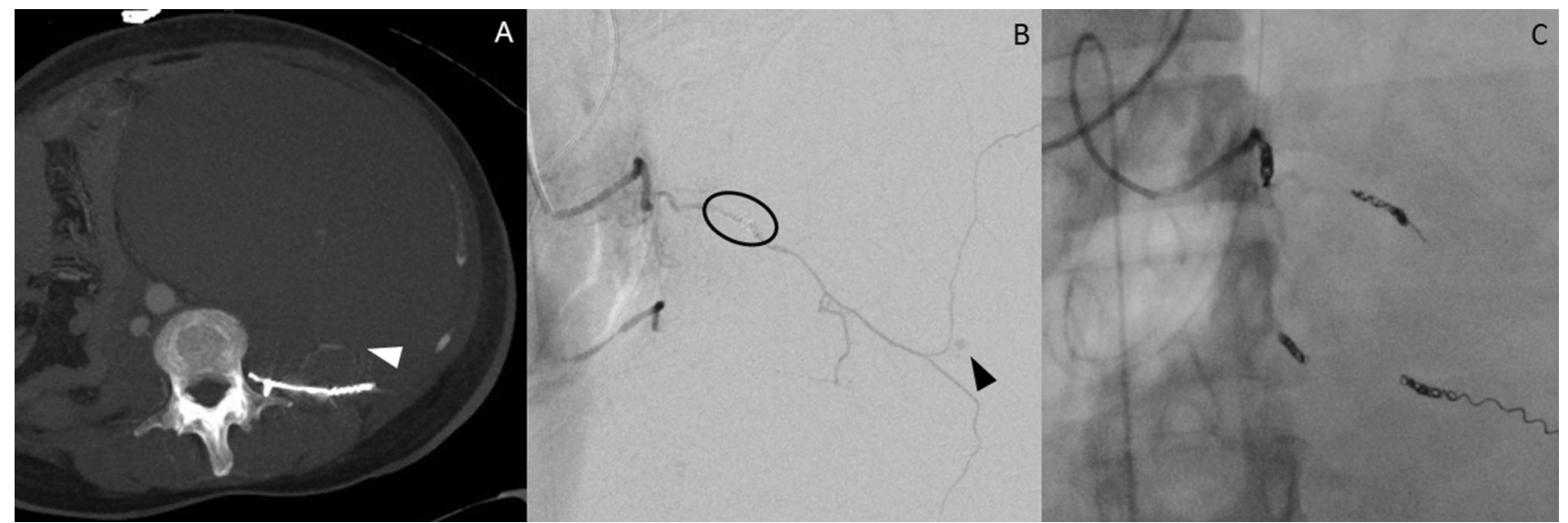

Fig.2 A CT scan of the same patient $3 \mathrm{~h}$ after TAE, performed due to worsening clinical condition ( $\mathrm{Hb}$ dropped to $5.1 \mathrm{~g} / \mathrm{dL}$ ), demonstrated significant enlarging of the hematoma and active blush from an embolized lumbar artery. B Angiography performed $4 \mathrm{~h}$ after the first

However in the remaining 7/21 patients, an attempt of conservative management was opted. After a median time of 2 days, all except one patient (6/7) were referred to angiography due to persistent $\mathrm{Hb}$ drop; moreover, $2 / 7$ died due to bleeding-related cardiovascular failure (28.6\%). This evidence supports that prompt angiographic management should be preferred.

Several technical aspects of TAE procedures should be underlined.

Superselective DSA depicted active blush only in 14/20 procedures; empiric embolization was performed based on the site of hematoma in $5 / 20$ procedure, and in $1 / 20$, no embolization was performed (requiring a second angiography 3 days later). These findings suggest that embolization should be performed even without sign of active bleeding.
TAE showed blood flow through the previously embolized lumbar artery (coils; circle) with sites of bleeding (harrow head). C Embolization was performed with coils and gelfoam, achieving sustained blood stasis, permitting patient recovery

TAE was performed with gelfoam alone $(4 / 19 ; 21 \%)$, gelfoam and coil $(12 / 19 ; 63.2 \%)$, and glue $(2 / 19 ; 10.5 \%)$. Coils were employed as a sole embolic agent in 1/19 procedure (5.3\%; Fig. 1). This patient rebled promptly requiring adjunctive TAE with coils and gelfoam (Fig. 2). This occurrence suggests adding embolic agents (gelfoam/ glue) in combination with coils to facilitate blood stasis and consequent clot formation. Moreover, during severe bleeding vasospasm may impede appropriate coil sizing and positioning; finally, multiple transfused patients are more likely to experience coagulation dysfunction [5] that could impair appropriate clot formation.

Concluding our impression on the interventional management of COVID-19 SSTH bleeding is that nearly all attempts to proceed with a conservative management failed, thus differing with non-COVID-19 SSTH; selective embolization should be performed even without sign of 
active bleeding; finally, adjunctive embolic agent (gelfoam /glue) employment is strongly suggested to facilitate clot formation.

Funding None.

\section{Compliance with Ethical Standards}

Conflict of Interest The authors declare that they have no conflict of interest.

\section{References}

1. Hippensteel JA, LaRiviere WB, Colbert JF, Langou t-Astri CJ, Schmidt EP. Heparin as a therapy for COVID-19: Current evidence and future possibilities. Am J Physiol—Lung Cell Mol
Physiol. 2020;319(2):211-7. https://doi.org/10.1152/AJPLUNG. 00199.2020 .

2. Dohan A, Darnige L, Sapoval M, Pellerin O. Spontaneous soft tissue hematomas. Diagn Interv Imaging. 2015;96(7-8):789-96. https://doi.org/10.1016/j.diii.2015.03.014.

3. Lucatelli P, De Rubeis G, Citone M, et al. Heparin-related major bleeding in Covid-19-Positive patient: perspective from the outbreak. Cardiovasc Intervent Radiol. 2020;43(8):1216-7. https://doi.org/10.1007/s00270-020-02532-3.

4. Touma L, Cohen S, Cassinotto C, et al. Transcatheter arterial embolization of spontaneous soft tissue hematomas: a systematic review. Cardiovasc Intervent Radiol. 2019;42(3):335-43. https:// doi.org/10.1007/s00270-018-2086-x.

5. Spahn DR, Bouillon B, et al. The European guideline on management of major bleeding and coagulopathy following trauma: fifth edition. Crit Care. 2019. https://doi.org/10.1186/ s13054-019-2347-3.

Publisher's Note Springer Nature remains neutral with regard to jurisdictional claims in published maps and institutional affiliations. 